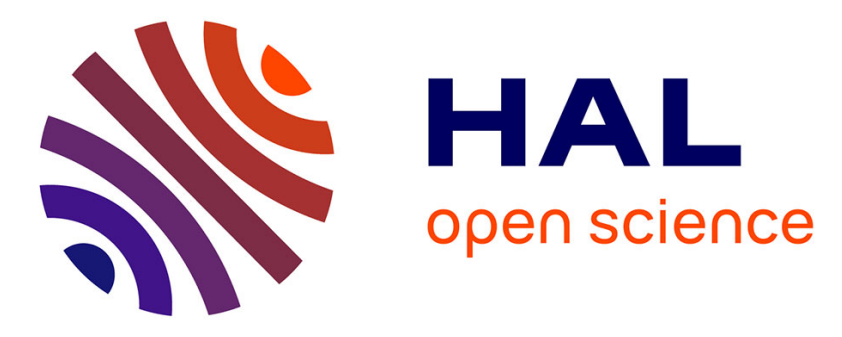

\title{
Time to Go Augmented in Vascular Interventional Neuroradiology?
}

\author{
René Anxionnat, Marie-Odile Berger, Erwan Kerrien
}

\section{To cite this version:}

René Anxionnat, Marie-Odile Berger, Erwan Kerrien. Time to Go Augmented in Vascular Interventional Neuroradiology?. Cristian Linte and Elvis Chen and Marie-Odile Berger and John Moore and David Holmes III. Augmented Environments for Computer-Assisted Interventions, 7815, Springer, pp.3-8, 2013, Lecture Notes in Computer Sciences, 10.1007/978-3-642-38085-3_2 . hal-00865726

\section{HAL Id: hal-00865726 \\ https://hal.inria.fr/hal-00865726}

Submitted on 25 Sep 2013

HAL is a multi-disciplinary open access archive for the deposit and dissemination of scientific research documents, whether they are published or not. The documents may come from teaching and research institutions in France or abroad, or from public or private research centers.
L'archive ouverte pluridisciplinaire HAL, est destinée au dépôt et à la diffusion de documents scientifiques de niveau recherche, publiés ou non, émanant des établissements d'enseignement et de recherche français ou étrangers, des laboratoires publics ou privés. 


\title{
Time to go augmented in vascular interventional neuroradiology?
}

\author{
René Anxionnat ${ }^{1}$, Marie-Odile Berger ${ }^{2,3}$, and Erwan Kerrien ${ }^{2,3}$ \\ 1 Therapeutic and Diagnostic Interventional Neuroradiology Dept, Univ. Hospital, \\ Nancy, F-54000, France \\ 2 Inria, Villers-lès-Nancy, F-54600, France, \\ 3 Université de Lorraine, Loria, UMR7503, Vandœuvre-lès-Nancy, F-54600, France
}

This editorial paper reports on our experience in introducing augmented reality $(\mathrm{AR})$ in interventional neuroradiology environments. Our expectations about the next AR tools, in particular for more advanced visualization, are also put forward. For practical reasons, the references will be restricted to our contributions. For further information, the last recommendations concerning the medical management of aneurysm induced hemorrhages can be found in [1].

\section{Interventional neuroradiology}

Interventional neuroradiology is a medical discipline that leverages minimally invasive techniques to operate on vascular lesions of the nervous system. The treatment is performed through the inside of the blood vessels, using medical imaging as visual feed-back. Such endovascular procedures are mainly performed to treat aneurysms. Intracranial aneurysms are sacciform ectasias of arteries located on the brain surface. The main associated risk is their rupture, leading to an hemorrhage with dramatic clinical consequences. Their size (diameter) varies between $2 \mathrm{~mm}$ and a few tens of millimeters. An endovascular treatment (EVT) progressively occludes the aneurysm with very soft spiral-like coils. EVTs were introduced in the 60's but really took off in the 90's after controlled detachable coils were developed. EVT has now replaced neurosurgery as the first choice treatment of aneurysms.

EVTs are performed in interventional vascular operating rooms where X-ray imaging enables the precise analysis of the lesions and their treatment. X-ray Angiography still remains the imaging modality of reference thanks to its optimal spatial and temporal resolution. The endovascular navigation followed by the EVT itself are performed under the real-time visual feed-back of a low dose and high frame rate ( $15 \mathrm{fps}$ on average) angiography image acquisition called fluoroscopy. However, these low quality images are projection images that do not contain any 3D information. Several viewpoints are required for an easier guidance through complex vascular networks and a better control of the treatment. Therefore aneurysm EVT are usually performed in biplane mode, providing simultaneous Antero-Posterior (AP) and Lateral (Lat) fluoroscopic views. No significant improvements were brought in the last few years in the control of EVTs using fluoroscopy. The development of Augmented Fluoroscopy very re- 
cently modified this status by introducing, for the first time, Augmented Reality in vascular interventional neuroradiology.

\section{Augmented Fluoroscopy: principle and clinical interest}

The idea of enriching fluoroscopy images with 3D data was born shortly after the development, in 1997, of 3D rotational angiography (3DRA) [2], a cone-beam tomography reconstruction techniques that produces a volume from angiography images acquired during a rotation of the vascular C-arm. 3DRA allows for an optimal treatment planning thanks to a precise analysis of the aneurysm morphology: its size, its shape and its neck, where it connects with its parent vessel [3]. From 3DRA, a working view can be defined that best identifies the aneurysmal neck and clearly reveals the limits for the coil positioning. 3DRA rapidly became a must-have imaging modality for EVT planning, to improve treatment safety and expand their indications. However, over a decade was necessary to actually integrate 3DRA with fluoroscopy thereby building the first augmented reality tool suitable for use in clinical practice.

During EVT, superimposing 3DRA onto the fluoroscopy sequence provides the physician with a stereoscopic vision of the morphology of vascular bifurcations, of the aneurysm and its relations with neighboring vessels. Endovascular guidance is made easier and coil deployment is better controlled.

Augmented Fluoroscopy (AF) is based on 3D/2D registration between 3DRA and fluoroscopy in any C-arm orientation. A prototype was developed in close collaboration between the research team (Inria Nancy Grand-Est), the manufacturer (GE Healthcare) and the medical staff (Department of Neuroradiology University Hospital of Nancy, France). The geometric accuracy of the implemented machine-based registration was first measured [4]. The next step consisted in further evaluating the prototype in the clinical conditions of an aneurysm EVT. Validation criteria were designed to address the clinical requirements met in the operating room, in terms of geometric accuracy and visualization. Visualization should indeed both preserve the visibility of the EVT tools (microcatheters, guidewires, and coils) and clearly depict the vascular topology in $3 \mathrm{D}$.

The geometric accuracy was first estimated on a silicon phantom of the cerebral vasculature under various orientations of the $\mathrm{C}$-arm. It was proved to be less than $0.5 \mathrm{~mm}$ and therefore compatible with the clinical objective [5]. This first conclusion was confirmed on clinical cases.

Concerning visualization, we chose to blend the 3DRA volume in Volume Rendering onto the fluoroscopic images. Easy to implement, this solution presents as a further advantage that it barely impacts the usual physician's environment (see Figure 1). Using AF in clinical conditions confirmed the relevance of this choice, and helped to significantly improve this prototype with functionalities such as interactive tuning of the vessel transparency to reach an optimal consensus between vascular relief and envovascular material visibility. After these successive improvements, AF was launched as a product in 2007. 

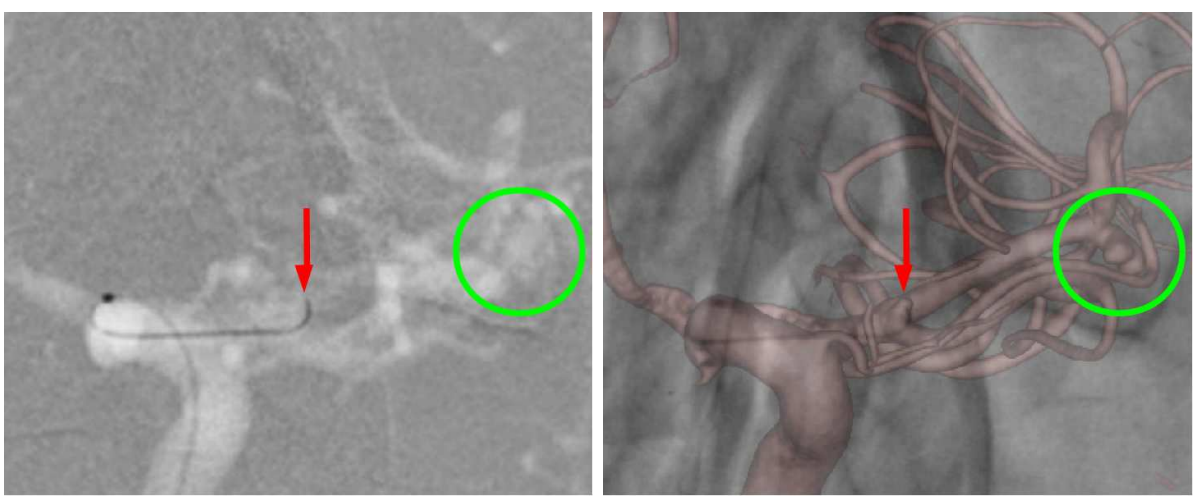

Fig. 1. Augmented Fluoroscopy (AF) case. (left) Original fluoroscopy image ; (right) $\mathrm{AF}$ view. AF offers a much clearer depiction of the ruptured aneurysm to be treated (green circle), and also unveils a microscopic incidental aneurysm which hampers the guide wire navigation (red arrow).

\section{The ordeal of going into clinical practice: an under-estimated step}

The medical team was involved in the development of AF right from the start, and the preliminary evaluation demonstrated its definite clinical interest. Nevertheless, several limitations made the integration of AF in clinical routine very gradual. First, AF is only available on the AP C-arm. Yet, in biplane mode, the working view used for the EVT may only be reached by the Lat C-arm. Second, in actual clinical practice, many events may cause the machine-based registration to degrade which quite often calls for a manual correction in the absence of an image-based registration adjustment.

Moreover, the AF monitor was first set aside in the operating room in order to minimize the perturbation on the otherwise unchanged environment. On one hand, the physicians readily accepted such a discrete modification in their work environment. On the other hand, the prototype was underutilized. Therefore, significant improvements, in terms of ergonomics, were made to better integrate the prototype into the operating room, such as larger zoom factors and an actual integration as part of the panel of control monitors.

The second step towards the use in clinical routine was gradual and more or less fast depending on physicians. Indeed, physicians needed some time to agree to change their work routine either during risky interventions that required a lot of concentration or for cases they could easily treat without AF. But this phase, including, and especially for the latter simple cases, proved essential to the evaluation by physicians who had to become familiar with the new environment and compare it with reference tools, namely casual fluoroscopy that was still available. Once AF was mastered, the physician could use it in complex cases and rely on information that would not be accessible with mere fluoroscopy, e.g. 
axial views of complex aneurysms. AF is being used in clinical routine at our institution since 2011.

\section{Perspectives: other applications of Augmented Fluoroscopy ; needs for further Augmented Reality tools}

AF marks a breakthrough in intraoperative visualization by introducing the third dimension. It was perfectly integrated into the EVT environment for intracranial aneurysms. Its use for other clinical applications and in combination with other 3D imaging modalities were logically pursued.

Arteriovenous malformations (AVMs) are scarcer than brain aneurysms and also differ a lot on both the morphology and therapy levels. They consist of a network of abnormal vessels interposed between arteries and veins which creates a high blood speed arteriovenous shunt in the brain parenchyma. The treatment is to occlude the AVM using fluid or pasty injections after a distal navigation within a very small, sub-millimetric, highly branched vascular network. Technological advances in the field of navigation tools and endovascular material allow to perform such embolization in very distal vascular areas, as well as highly functional brain regions.

AF can facilitate this complex navigation by displaying the origin and path of afferent arteries and differentiating them from neighboring vessels or vessels that superimpose in the fluoroscopic view. These clinical needs are more demanding than for aneurysms given the complexity and finesse of the vascular network. Additional work in terms of accuracy of registration, and vascular segmentation and visualization will be necessary to make this tool functional in clinical practice.

During an EVT, the physician can only see vessels on the monitors, but has no access to a direct visualization of the cerebral parenchyma in the surroundings of the endovascular material. Locating crucial functional brain areas cannot be realized but through a mental effort to relate a priori anatomical knowledge and preoperative imaging to intraoperative views. The inherent inaccuracy of such process may lead the physician to excessively embolize in highly functional areas, potentially causing dramatic complications, or, conversely to give up treatment in case of doubt. A multimodal localization is definitely required, especially combining MRI and angiography, to identify the cerebral regions with a high accuracy. Innovative visualization tools will have to be developed to setup such new multimodality environments and bring relevant information, in an intuitive way, to the physician during the treatment.

During the treatement, AF provides $3 \mathrm{D}$ vascular information in any orientation the C-arm can reach. As such, a real progress was made compared to mere fluoroscopy imaging. However, endovascular material still is only visible under projection in limited orientations. The next step will see the 3D reconstruction of endovascular tools and material during the EVT. These tools and material will be directly located in the 3DRA volume and thereby, on one hand, visible in 
any view, including virtual views, such as axial views inaccessible to the $\mathrm{C}$-arm, and on the other hand, easy to relate to any $3 \mathrm{D}$ multimodal imaging volume. Very hard technical and scientific issues will have to be overcome, but it casts a glimpse of potentially dramatic breakthroughs in the way EVTs are performed.

Having such a possibility will allow for a better control of the EVT, thus improving safety. However working in a multimodal environment will generate inaccuracies due to the involved registration and segmentation processes, that will need to be quantified and that the physician should be fully aware of. The physician will have to agree to cope with this uncertainty when working on virtual views without the direct control provided by fluoroscopy, and thus no possibility for correction. An important intellectual investment will be necessary to learn how to use these tools and manage this uncertainty before appropriating these tools in clinical practice.

Eventually, future augmented environments will be enriched not only with visual information from other imaging modalities such as functional, but also with other data such as generated by hemodynamic simulation or wall pressure computations for aneurysms.

\section{Conclusion}

Our experience with AF shows it is possible to develop Augmented Reality tools in the field of interventional neuroradiology. But there is a long way between the initial idea and the product used in clinical practice: 10 years in the case of AF.

For this tool to be used it must meet specific clinical needs, clearly identified by physicians, and be validated for this purpose. This validation must meet specific criteria and objectives. In the case of an EVT, which corresponds to a risky procedure requiring a high concentration from the physician, any Augmented Reality tool must be fully integrated into the operating room and provide an intuitive interface.

It is important that any operator that was not necessarily involved in the development and validation process, be persuaded to use the tool first in simple cases where it can be evaluated within a personal operating environment so as to gradually become more familiar with the tool and finally trust the additional information it provides. Under these conditions the augmented environment has the potential to bring real benefits to the physician, especially in complex cases, and imply actual changes in the clinical practice. As an example, AF provides actual 3D information which biplane fluoroscopy useless for most interventions and thus reduceing patient irradiation and exam time while probably improving the safety of the intervention.

AF has paved the way for Augmented Reality in interventional neuroradiology. A vast field of opportunities exist to adapt this tool to other, more demanding, clinical applications and develop new Augmented Reality tools. New angiographic rooms will emerge that integrate an augmented environment, allowing the interventional neuroradiologist to work in fully multimodal setup enriched with visual, and other kinds of, information. 


\section{References}

1. Connolly, E.S., J., Rabinstein, A., Carhuapoma, J.R., Derdeyn, C.P., Dion, J., Higashida, R., Hoh, B., Kirkness, C., Naidech, A., Ogilvy, C., Patel, A., Thompson, B., Vespa, P.: Guidelines for the management of aneurysmal subarachnoid hemorrhage. Stroke 43 (2012) 1711-1737 DOI: 10.1161/STR.0b013e3182587839.

2. Anxionnat, R., Bracard, S., Macho, J., da Costa, E., Vaillant, R., Launay, L., Trousset, Y., Romas, R., Picard, L.: 3D angiography. clinical interest. first applications in interventional neuroradiology. J. Neuroradiol. 25(4) (1998) 251-262

3. Anxionnat, R., Bracard, S., Ducrocq, X., Trousset, Y., Launay, L., Kerrien, E., Braun, M., Vaillant, R., Scomazzoni, F., Lebedinsky, A., Picard, L.: Intracranial aneurysms: Clinical value of 3D digital subtraction angiography in the therapeutic decision and endovascular treatment. Radiology 218(3) (2001) 799-808

4. Gorges, S., Kerrien, E., Berger, M.O., Trousset, Y., Pescatore, J., Anxionnat, R., Picard, L.: Model of vascular C-arm for 3D augmented fluoroscopy in interventional radiology. In Duncan, J., Gerig, G., eds.: Proc. of MICCAI'05. Volume 3750 of LNCS., Springer-Verlag (2005) 214-222 DOI: $10.1007 / 11566489 \_27$.

5. Gorges, S., Kerrien, E., Berger, M.O., Pescatore, J., Trousset, Y., Anxionnat, R., Bracard, S., Picard, L.: 3D augmented fluoroscopy in interventional neuroradiology: precision assessment and first evaluation in clinical cases. In: Proc. of AMI-ARCS'06. (2006) 